\title{
Effect of Ethanol on Fluoroquinolone Efficacy in a Rat Model of Pneumococcal Pneumonia
}

\author{
Keith M. Olsen, ${ }^{1}$ Martha Gentry-Nielsen, ${ }^{2,3,4,5}$ Mei Yue, ${ }^{4}$ Mary U. Snitily, ${ }^{4}$ \\ and Laurel C. Preheim ${ }^{2,3,4,5 *}$ \\ Colleges of Pharmacy ${ }^{1}$ and Medicine, ${ }^{2}$ University of Nebraska Medical Center, 600 South 42nd St., Omaha, Nebraska 68198; \\ Infectious Diseases Section, Veterans Affairs Medical Center, ${ }^{3}$ and Veterans Affairs Medical Center ${ }^{4}, 4101$ Woolworth Avenue, \\ Omaha, Nebraska 68105; and Creighton University School of Medicine, 2500 California Plaza, Omaha, Nebraska $68178^{5}$
}

Received 13 September 2004/Returned for modification 13 November 2004/Accepted 26 September 2005

\begin{abstract}
This investigation compared the effect of ethanol on fluoroquinolone antibiotic efficacy and pharmacodynamics in an ethanol-fed rat model of pneumococcal pneumonia. Male Sprague-Dawley rats received a liquid diet containing $36 \%$ of total calories as ethanol. Paired controls (pair-fed controls) were fed a liquid diet without ethanol or received rat chow. Diets began 7 days before and continued for 10 days after transtracheal infections with 10 times the $50 \%$ lethal dose of type 3 Streptococcus pneumoniae. Beginning $18 \mathrm{~h}$ after infection, the rats received once daily subcutaneous phosphate-buffered saline, levofloxacin, moxifloxacin, or trovafloxacin at 50 or $100 \mathrm{mg} / \mathrm{kg}$ of body weight. White blood cell counts were determined, blood samples were collected for culture, and mortality was recorded. Additional rats were killed on day 5 for pharmacodynamic studies and quantitative cultures of bronchoalveolar lavage fluid. Bacteremia occurred by day 3 in 20 of 22 untreated rats. All 22 untreated rats died by day 9. Moxifloxacin treatment was effective in all diet groups at both the 50- and $100-\mathrm{mg} / \mathrm{kg}$ doses. In contrast, $50-\mathrm{mg} / \mathrm{kg}$ doses of levofloxacin and trovafloxacin improved survival in ethanol-fed rats but were ineffective in chow-fed rats. High-dose trovafloxacin at $100 \mathrm{mg} / \mathrm{kg}$ was associated with increased mortality in pair-fed rats. The free-fraction area under the concentration-time curve/MIC ratio exceeded 50 with all antibiotics in the ethanol group but dropped below 30 with levofloxacin and trovafloxacin in the pairand chow-fed rats, with higher mortality. Achievement of adequate antibiotic-free fraction area under the concentration-time curve/MIC ratios helps overcome ethanol-induced immune defects induced in experimental pneumococcal pneumonia.
\end{abstract}

Community-acquired pneumonia (CAP) is a common respiratory infection associated with significant morbidity and mortality $(21,35)$. Death rates in patients with CAP have not changed significantly over the past 20 years $(5,21)$. In those subjects who become bacteremic, mortality may exceed $25 \%$ in some patient populations $(25,37,61)$. Mortality is high among the elderly and in patients with underlying comorbidities, such as congestive heart failure, coronary artery disease, malignancy, and ethanol (EtOH) abuse $(5,25,35,41)$. Although numerous pathogens have been associated with CAP, empirical antimicrobial therapy is directed against a small number of organisms. Streptococcus pneumoniae, or the pneumococcus, is the most common cause of CAP in all patient populations, regardless of severity, and is responsible for two-thirds of bacteremic cases (1).

Alcoholism affects up to 8 to $10 \%$ of men and 1 to $2 \%$ of woman, placing them at high risk for medical complications (34). EtOH abuse decreases host defenses against pneumonia and increases the risk of severe pneumococcal infections (13, 33). Using an EtOH-fed rat model, we previously demonstrated that polymorphonuclear leukocytes (PMNLs) have an impaired ability in vitro to kill $S$. pneumoniae $(23,26,27)$. This EtOH-induced defect in PMNL function is not due to a reduction in phagocytic activity but is related to the diminished

* Corresponding author. Mailing address: VA Medical Center, 4101 Woolworth Avenue, Omaha, NE 68105. Phone: (402) 449-0650. Fax: (402) 977-5602. E-mail: laurel.preheim@med.va.gov. production of oxygen free radicals and decreased granulation (27). EtOH ingestion has also been shown to induce glycosylation of iron-binding proteins important for withholding iron from invading pathogens, resulting in a diminished inhibitory effect on microorganism growth (57). In addition, acute EtOH ingestion down-regulates the production and release of the proinflammatory mediator tumor necrosis factor (29). These EtOH-induced effects on PMNLs, iron binding, and the inflammatory response markedly impair host defenses against invasive $S$. pneumoniae $(23,36,41,42,43)$.

Fluoroquinolones have excellent in vitro activities and in vivo efficacies against many respiratory pathogens, including multidrug-resistant $S$. pneumoniae $(8,28,48,49)$. Therapeutic guidelines generally list the advanced quinolones levofloxacin and moxifloxacin as first-line therapies for CAP complicated by the presence of comorbidities in both ambulatory and hospitalized patients $(5,21,35)$. The pharmacokinetics of levofloxacin and moxifloxacin differ in that levofloxacin is predominantly eliminated renally, while the major elimination route for moxifloxacin is hepatic metabolism by conjugation $(14,15)$. Alatrofloxacin is an L-alanyl-L-alanyl prodrug which is rapidly converted to the parent fluoroquinolone, trovafloxacin $(9,56)$.

Trovafloxacin has activity against multidrug-resistant pneumococci $(19,30,54)$ and shares a similar route of hepatic elimination with moxifloxacin. We have previously demonstrated in an experimental model of hepatic cirrhosis that trovafloxacin has excellent efficacy, with superior area under the serum concentration-time curve (AUC)/MIC and maxi- 
Feeding Groups

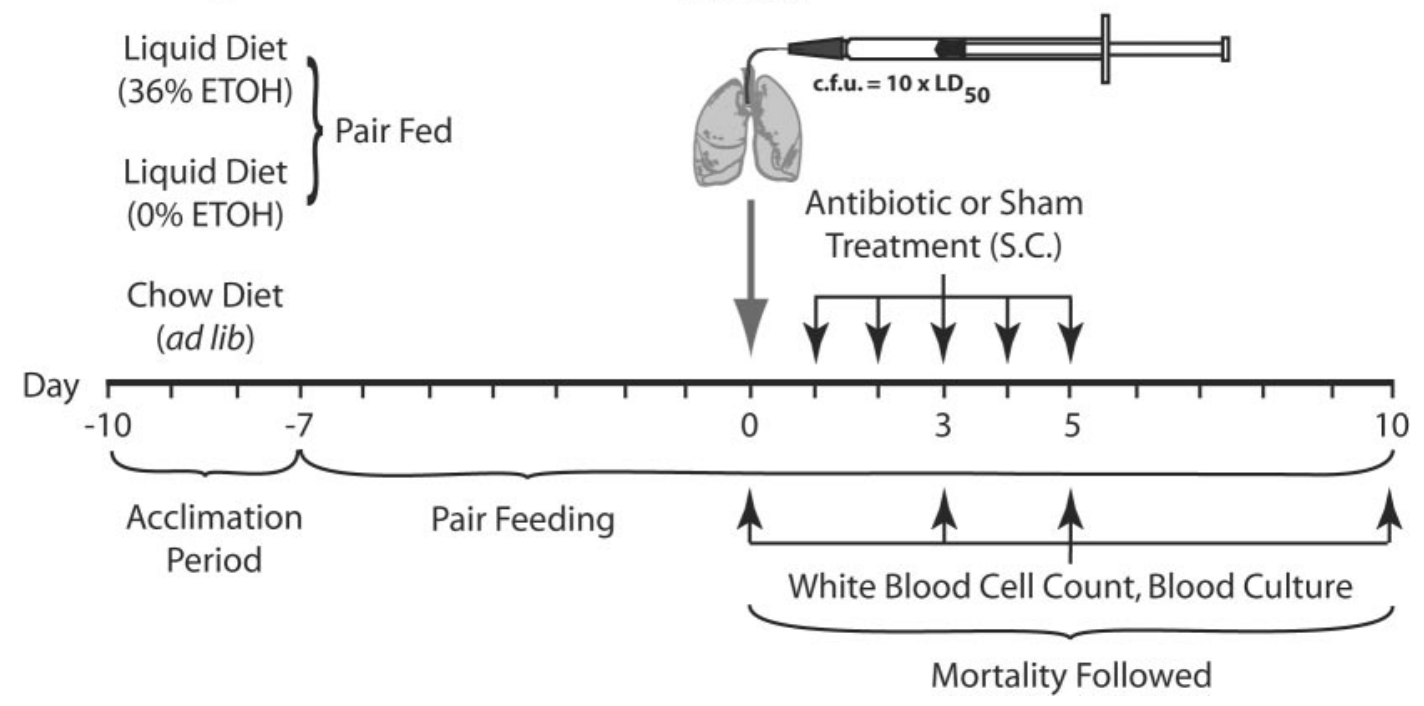

FIG. 1. Animal feeding groups and experimental timeline. $\mathrm{LD}_{50}=50 \%$ lethal dose; S.C., subcutaneous. mum concentration of drug in serum $\left(C_{\max }\right) / \mathrm{MIC}$ pharmacodynamic ratios compared to those of either a macrolide or a beta-lactam antibiotic (45).

Antibiotic pharmacodynamics are important predictors of the therapeutic response rate in experimental pneumococcal infection models $(20,24,32,38)$. However, the impact of EtOH on antibiotic efficacy and pharmacodynamic parameters remains unknown. The purpose of this study was to determine (i) the impact of EtOH on fluoroquinolone efficacy in an experimental model of pneumococcal pneumonia; (ii) the effect of EtOH on serum, intrapulmonary, and macrophage fluoroquinolone concentrations; and (iii) the influence of EtOH on fluoroquinolone pharmacodynamic parameters and their relationship to efficacy.

\section{MATERIALS AND METHODS}

Chronic EtOH ingestion model. Male Sprague-Dawley rats (Charles River Laboratories, Kingston, NY) were fed standard rat chow and allowed water ad libitum until they reached an average weight of $225 \mathrm{~g}$. EtOH- and pair-fed rats were then switched to nutritionally adequate liquid diets (Dyets, Bethelehem, PA). The EtOH-fed rats received $36 \%$ of their total calories as EtOH. Isocaloric dextrin-maltose replaced EtOH in the liquid control diet. Rats from each liquid diet group were matched in pairs by similar weight. Within each pair, one rat was fed an amount of control diet equal to the amount of EtOH diet consumed by its EtOH-fed pair on the previous day (this is referred to as the pair-fed control group). Rats were pair fed in this manner for a period of 7 days before infection with $S$. pneumoniae (Fig. 1). A third group of chow-fed rats served as controls and were maintained on standard rat chow and water ad libitum (22). Approval was received from the Omaha V.A. Animal Care and Use Committee prior to initiation of this study.

Experimentally induced pneumococcal pneumonia. Type 3 S. pneumoniae (ATCC 6303; American Type Culture Collection, Manassas, VA) was grown to logarithmic phase in Todd-Hewitt broth containing 5\% rabbit serum. The organisms were collected by centrifugation, washed twice, and suspended in phosphate-buffered saline (PBS). The three experimental groups, the chow-fed control rats, the EtOH-fed rats, and the pair-fed rats, were anesthetized with isoflurane (Halocarbon Laboratories, River Edge, NJ). A small incision was made in the skin to surgically expose the trachea (22). A 20-gauge catheter was inserted into the main-stem bronchus, through which $0.3 \mathrm{ml}$ of the pneumococcal suspension containing $3 \times 10^{7} \mathrm{CFU}$ was injected transtracheally for pair- and chow-fed rats and $3 \times 10^{6} \mathrm{CFU}$ was injected for EtOH-fed rats (36). These quantities of organisms represent 10 times the $50 \%$ lethal dose for the respective groups of animals, such that all untreated animals would be expected to die (42).

Antimicrobial treatment. The dosages of levofloxacin, moxifloxacin, and trovafloxacin were selected to approximate the $C_{\max }$ and AUC to those achieved in humans $(7,40,49,50,54)$. The free-fraction AUC (fAUC) approximated that found from studies with humans. Groups of 12 rats from each feeding group were evenly divided and given daily subcutaneous doses of 50 or $100 \mathrm{mg} / \mathrm{kg}$ of body weight of levofloxacin (Ortho-McNeil Pharmaceutical, Inc., Raritan, NJ), moxifloxacin (Bayer Corp., West Haven, CT), or trovafloxacin (Pfizer Pharmaceuticals, Inc., Groton, CT). A separate group of six rats from each feeding group was sham treated with PBS. All rats were treated for 5 days, beginning at $18 \mathrm{~h}$ postinfection (Fig. 1). The $S$. pneumoniae MICs of the study antibiotics for the infecting strain, determined with Etest strips, were as follows: levofloxacin, 1.0 $\mu \mathrm{g} / \mathrm{ml}$; moxifloxacin, $0.125 \mu \mathrm{g} / \mathrm{ml}$; and trovafloxacin, $0.19 \mu \mathrm{g} / \mathrm{ml}$.

Peripheral WBC counts, blood cultures, and efficacy studies. Survival was monitored for 10 days postinfection, with blood samples for quantitative culture obtained by aseptic foot puncture (51) on day 1 (pretreatment) and days 3, 5, and 10 postinfection (Fig. 1). The number of bacteria present per $\mathrm{ml}$ of blood was determined by standard plate counting technique.

White blood cell (WBC) counts prior to infection and on days 3, 5, and 10 after infection were determined with a hemacytometer. Differential cell counts were determined on stained slides (Diff-Quik; Baxter Scientific Products, McGraw Park, IL).

BAL. Rats (seven per group) not used in the mortality studies were infected and treated as described above. On day 3,1 h following administration of the third antibiotic dose, rats from each antibiotic dose group were killed by an intraperitoneal injection of pentobarbital (Nembutal; Abbott Laboratories, North Chicago, IL) and exsanguinated by cardiac puncture. Bronchoalveolar lavage (BAL) was performed with cold PBS to produce $50 \mathrm{ml}$ of BAL fluid from each rat, as described previously (44). Aliquots of BAL fluid were cultured quantitatively by plate counts. Cells in the BAL fluid were then collected by centrifugation, resuspended in PBS, and counted with a hemacytometer. Differential counts were performed on Wright-stained cytospin slides of the cell preparations.

Samples for pharmacokinetic and pharmacodynamic analyses. Blood samples for determination of the values of pharmacokinetic and pharmacodynamic parameters were obtained by cardiac puncture from separate groups of rats $(n=6)$ not used in the mortality studies. On the 5th day of therapy and after administration of the fifth antibiotic dose, blood samples were obtained at 1, 3, 5, 12, and 24 h. All blood samples were transferred to red-top tubes (Vacutainer; BectonDickinson, Franklin Lakes, NJ), allowed to clot for $20 \mathrm{~min}$ at room temperature, and centrifuged at $2,000 \times g$ for $10 \mathrm{~min}$. All serum samples were frozen at $-80^{\circ} \mathrm{C}$ for antibiotic concentration determination. 

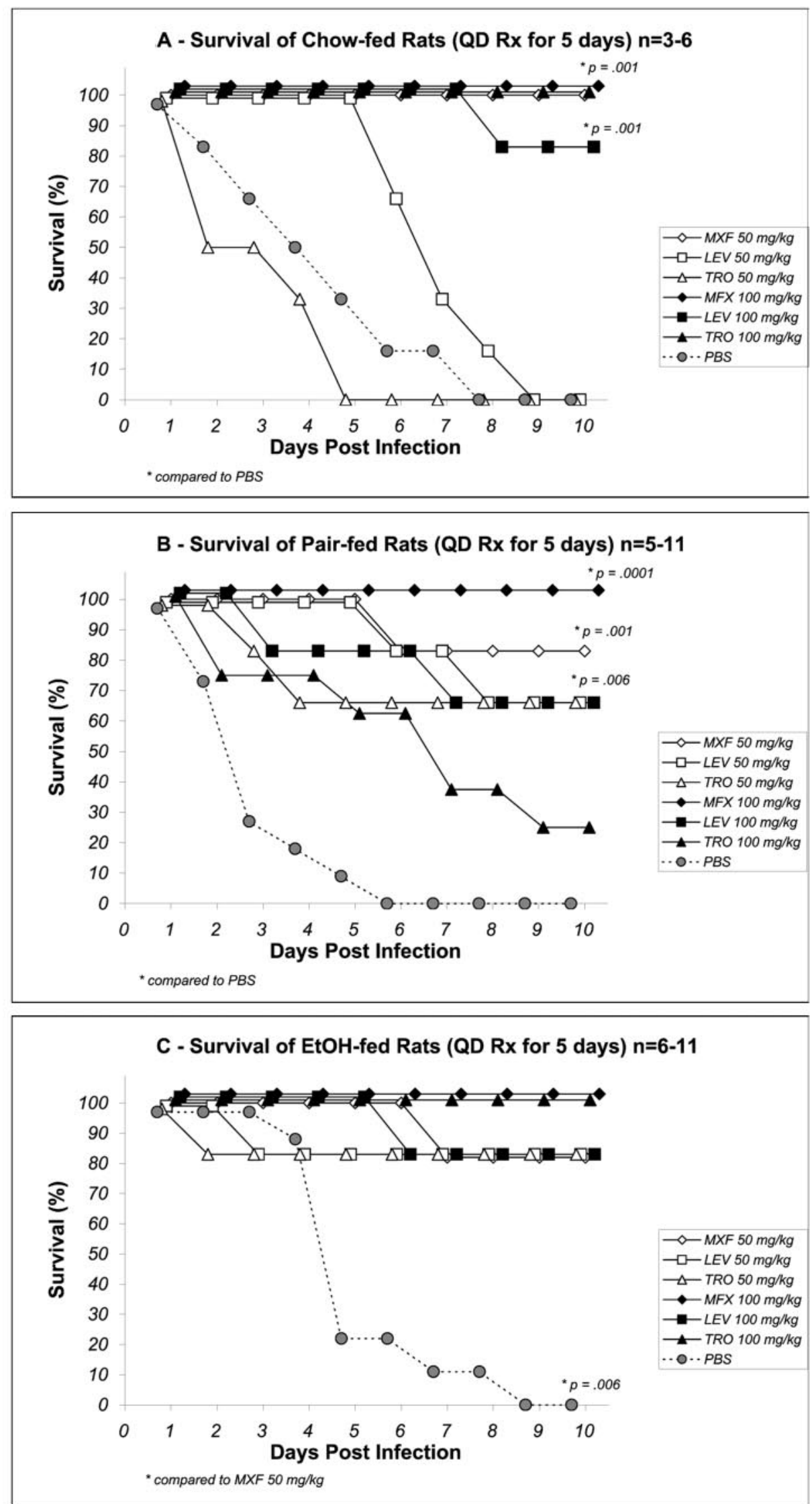

FIG. 2. Cumulative percent survival after 5 days of antibiotic therapy in chow-fed (A), pair-fed (B), and EtOH-fed (C) rats. All antibiotics were administered at doses of 50 and $100 \mathrm{mg} / \mathrm{kg} /$ day. LEV, levofloxacin; MXF, moxifloxacin; TRO, trovafloxacin. *, $P$ values compared to the results for moxifloxacin $50 \mathrm{mg} / \mathrm{kg}$ or to PBS as indicated. 
Concentration of antibiotics in serum, BAL fluid, and BAL cells. The concentrations of levofloxacin in BAL fluid, BAL cells, and serum were determined by modifications of previously published assays with UV detection $(12,60)$. BAL cells were lysed by freeze-thaw cycles. Acetonitrile containing the internal standard (ciprofloxacin; Bayer Corp.) was added to the cells for deproteination. The samples were then vortexed and centrifuged before $50 \mu$ l of the supernatant was injected onto the column. The BAL fluid samples were centrifuged and then filtered through a $0.22-\mu \mathrm{m}$-pore-size filter to remove the bacteria prior to extraction and injection onto the column. Following deproteination, BAL fluid samples were evaporated to dryness and reconstituted with the mobile phase prior to injection onto the column. Separation was performed with a $\mathrm{C}_{18}$ reversephase column (Phenomix, Torrance, $\mathrm{CA}$ ) at $50^{\circ} \mathrm{C}$ with $\mathrm{UV}$ detection set at 280 $\mathrm{nm}$. The mobile phase consisted of acetonitrile-1 mM 1-octanesulfonic acid (45:55 [vol/vol]; $\mathrm{pH} 3.5)$ at a flow rate of $1.5 \mathrm{ml} / \mathrm{min}$. Quality control samples were included in each analytical sequence to confirm the accuracy and precision of the assay, which was linear over the range of 0.1 to $20 \mu \mathrm{g} / \mathrm{ml}$, with the limit of quantification set at $0.1 \mu \mathrm{g} / \mathrm{ml}$. Samples with concentrations that exceeded the maximum linear concentrations were diluted and reinjected onto the column. The inter- and intraday coefficients of variation were 3.7 and $4.9 \%$, respectively, for serum; 7.5 and $8.2 \%$, respectively, for BAL cells; and 7.7 and $9.3 \%$, respectively, for BAL fluid. The volume of BAL cells from the cell pellet was determined by methods described previously (39).

The moxifloxacin concentrations in all matrixes were prepared in a similar manner and were determined by a previously published assay (50). This assay used solid-phase extraction and a reverse-phase chromatography method with fluorescence detection set at $296 \mathrm{~nm}$. Prior to extraction, the BAL cells were processed as described above. The proteins in the matrixes were removed by precipitation with mobile phase, placed in a shaking ultrasonic water bath for 10 min, and centrifuged. The supernatant was collected and evaporated to dryness for BAL fluid and BAL cells and reconstituted with mobile phase. Following the extraction procedure, the supernatants of all matrixes containing the internal standard (ciprofloxacin) were separated by a $\mathrm{C}_{18}$ analytical column and guard column (Phenomix) at $45^{\circ} \mathrm{C}$. The mobile phase consisted of a mixture of acetonitrile and tetrabutylammonium hydrogen sulfate solution (40:60 [vol/vol]; pH 3.0). The lower range of quantification was $1.0 \mu \mathrm{g} / \mathrm{ml}$ and was linear over the range of 5 to $50 \mu \mathrm{g} / \mathrm{ml}$ for all matrixes. The intra- and interday coefficients of variation at $1.0 \mu \mathrm{g} / \mathrm{ml}$ were 3.9 and $4.8 \%$, respectively, for serum; 6.7 and $9.3 \%$, respectively, for BAL cells; and 6.1 and $9.0 \%$, respectively, for BAL fluid.

The trovafloxacin concentrations in all matrixes were determined by a previously published assay $(52,53)$. This assay used solid-phase extraction and a reverse-phase chromatography method with UV detection. Prior to extraction, the BAL cells were exposed to freeze-thaw cycles to release intracellular drug. Following the extraction procedure, the supernatants of all matrixes containing the internal standard (ciprofloxacin) were separated by a $\mathrm{C}_{18}$ analytical column and equivalent guard column (Phenomix), with the wavelength set at $275 \mathrm{~nm}$. The mobile phase consisted of $0.04 \mathrm{M} \mathrm{H}_{3} \mathrm{PO}_{4}$-acetonitrile-tert-butylammonium hydroxide-0.005 $\mathrm{M}$ dibutyl amine phosphate reagent (82:16.9:0.05:0.05 [vol/vol]) at $\mathrm{pH}$ 3. The lower range of quantification was $0.1 \mu \mathrm{g} / \mathrm{ml}$ and was linear over the range of 0.1 to $20 \mu \mathrm{g} / \mathrm{ml}$ for all matrixes. The intra- and interday coefficients of variation at $1.0 \mu \mathrm{g} / \mathrm{ml}$ were 3.9 and $4.8 \%$, respectively, for serum; 6.5 and $8.3 \%$, respectively, for BAL cells; and 5.9 and $8.1 \%$, respectively, for BAL fluid.

Albumin and urea concentrations in serum and BAL fluid were determined by a previously described method (39). The volume of epithelial lining fluid (ELF) contained in each BAL fluid sample was determined by the urea dilution method, and the results were confirmed by application of the same method with albumin dilution (47). The ELF volume $\left(V_{\mathrm{ELF}}\right)$ is estimated by the following relationship: $V_{\mathrm{BAL}} \times\left(\right.$ urea $_{\mathrm{BAL}} /$ urea $\left._{\text {serum }}\right)$, where $V_{\mathrm{BAL}}$ is the volume of BAL fluid, urea $\mathrm{BAL}$

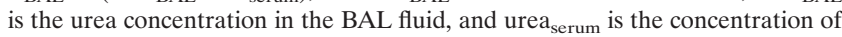
urea in the serum. The concentration of antibiotic in the ELF (Abx $\mathrm{ELF}_{\mathrm{EL}}$ ) was determined by the relationship $\mathrm{Abx}_{\mathrm{BAL}} / V_{\mathrm{ELF}}$, where $\mathrm{Abx}_{\mathrm{BAL}}$ is the total amount of drug in each BAL fluid sample. The volume of BAL cells in the cell pellet was determined by cytocentrifugation and multiplication of the mean cell volume and the total number of BAL cells. The mass concentration of antibiotic divided by the cell volume produced a concentration in micrograms of cell volume $(3,58)$.

For determination of protein binding, aliquots of serum were adjusted to a $\mathrm{pH}$ of 7.4 when necessary prior to separation. Separation of the free fractions from the protein-bound fractions of levofloxacin, moxifloxacin, and trovafloxacin was performed by ultrafiltration techniques. Briefly, $0.375 \mathrm{ml}$ of serum was divided into two aliquots, with one aliquot inserted into the ultrafiltration device (Amicon, Inc. Beverly, MA) and centrifuged at a fixed angle at $1,000 \times g$ for $20 \mathrm{~min}$. The nonfiltered serum aliquot and the resultant ultrafiltrate were analyzed for each antibiotic. The ratio of the resultant concentrations was then used to calculate the antibiotic free fraction. To determine the impact of the concentration on fluoroquinolone protein binding, serum aliquots were spiked with levofloxacin, moxifloxacin, and trovafloxacin at concentrations of 5, 1015,20 , and $25 \mu \mathrm{g} / \mathrm{ml}$ and analyzed as described above. Over the study concentrations and similar to the concentrations observed in the study with animals, no significant variance in protein binding that would have influenced the final fAUC/MIC calculations was detected.

Pharmacokinetic and pharmacodynamic analyses. Antibiotic serum concentration-versus-time data were analyzed with WinNonlin software (standard edition, version 1.5; Scientific Consulting, Inc., Cary, NC). The pharmacokinetic parameters for the fluoroquinolone antibiotics were estimated by using a noncompartmental extravascular dose input model. The AUC from time zero to $24 \mathrm{~h}$ $\left(\mathrm{AUC}_{0-24}\right)$ was calculated by the trapezoidal rule. Pharmacodynamic parameters were determined by dividing the MIC of each antibiotic for S. pneumoniae 6303 into the fAUC (fAUC/MIC) and $C_{\max }\left(C_{\max } / \mathrm{MIC}\right)$. The actual $C_{\max }$ was determined by extrapolating the time curve back to a time just after administration by using the equation $C_{\min }=C_{\max } e^{- \text {ket }}$, where $C_{\min }$ equals the minimum concentration of drug in the serum, $C_{\max }$ equals the calculated maximum peak serum concentration, and $e^{- \text {ket }}$ is the decay parameter.

Statistical analysis. Differences in the development of bacteremia and the mortality studies were analyzed by Fisher's exact test. The number of white blood cells and bacteria in the BAL fluid samples were compared by one-way analysis of variance, with post-hoc comparisons by Tukey's test. The mean values of the pharmacokinetic and pharmacodynamic parameters were compared by one-way analysis of variance, with post-hoc comparisons by Newman-Keuls test. All mean data are reported as means \pm standard deviations. The level of significance was set at a $P$ value of $<0.05$ for all analyses.

\section{RESULTS}

Survival studies. In all diet groups combined, 20 of 22 PBS sham-treated rats were bacteremic by day 3 , and all 22 had died by day 9 . In chow-fed rats, levofloxacin and trovafloxacin were ineffective at a dose of $50 \mathrm{mg} / \mathrm{kg}$ (Fig. 2A), whereas all animals treated with the same dose of moxifloxacin survived $(P<0.05)$. At the higher dose of $100 \mathrm{mg} / \mathrm{kg}$, all study antibiotics were equally effective in chow-fed rats (Fig. 2A). In pair-fed rats, levofloxacin and trovafloxacin were more effective at the 50$\mathrm{mg} / \mathrm{kg}$ dose than the PBS sham treatment. The higher dose did not improve survival and was associated with an increased mortality in the trovafloxacin treatment group (Fig. 2B). Moxifloxacin was equally effective in pair-fed rats at either the 50- or the $100-\mathrm{mg} / \mathrm{kg}$ dose, with 11 of 12 rats surviving $(P<0.05$ versus the results for levofloxacin and trovafloxacin; $50-\mathrm{mg} / \mathrm{kg}$ regimens). The 10-day survival in EtOH-fed rats was equivalent with all antibiotics at both the 50 - and the $100-\mathrm{mg} / \mathrm{kg}$ doses with 10 of 12, 10 of 12, and 11 of 12 rats in the levofloxacin, moxifloxacin and trovafloxacin treatment groups, respectively, surviving (Fig. 2C).

Bacteremia and organisms in BAL fluid. Among the chowfed rats, fewer rats were bacteremic during or immediately after treatment, but by day 10 all PBS treated rats had died (Fig. 3A). All rats in the pair-fed and EtOH-fed groups receiving PBS treatment were bacteremic by day 3 , and blood cultures remained positive for the duration of the study or until death (Fig. 3B and C). Among the chow-fed rats, the $50-\mathrm{mg} / \mathrm{kg}$ levofloxacin and trovafloxacin regimens were least effective in clearing the bacteremia at all time points (Fig. 3A). Only moxifloxacin at $50 \mathrm{mg} / \mathrm{kg} /$ day and $100 \mathrm{mg} / \mathrm{kg} /$ day maintained sterility at all time points in this group. In contrast, the EtOHfed groups treated with moxifloxacin or trovafloxacin at 100 $\mathrm{mg} / \mathrm{kg}$ /day maintained blood sterility through day 10 (Fig. 3C). EtOH-fed rats that received moxifloxacin and trovafloxacin at $50 \mathrm{mg} / \mathrm{kg} /$ day had negative blood cultures by day 5 , but at day 10 regrowth was demonstrated in one of six $(17 \%)$ and two of six $(33 \%)$ rats, respectively (Fig. 3C). Among the EtOH-fed 


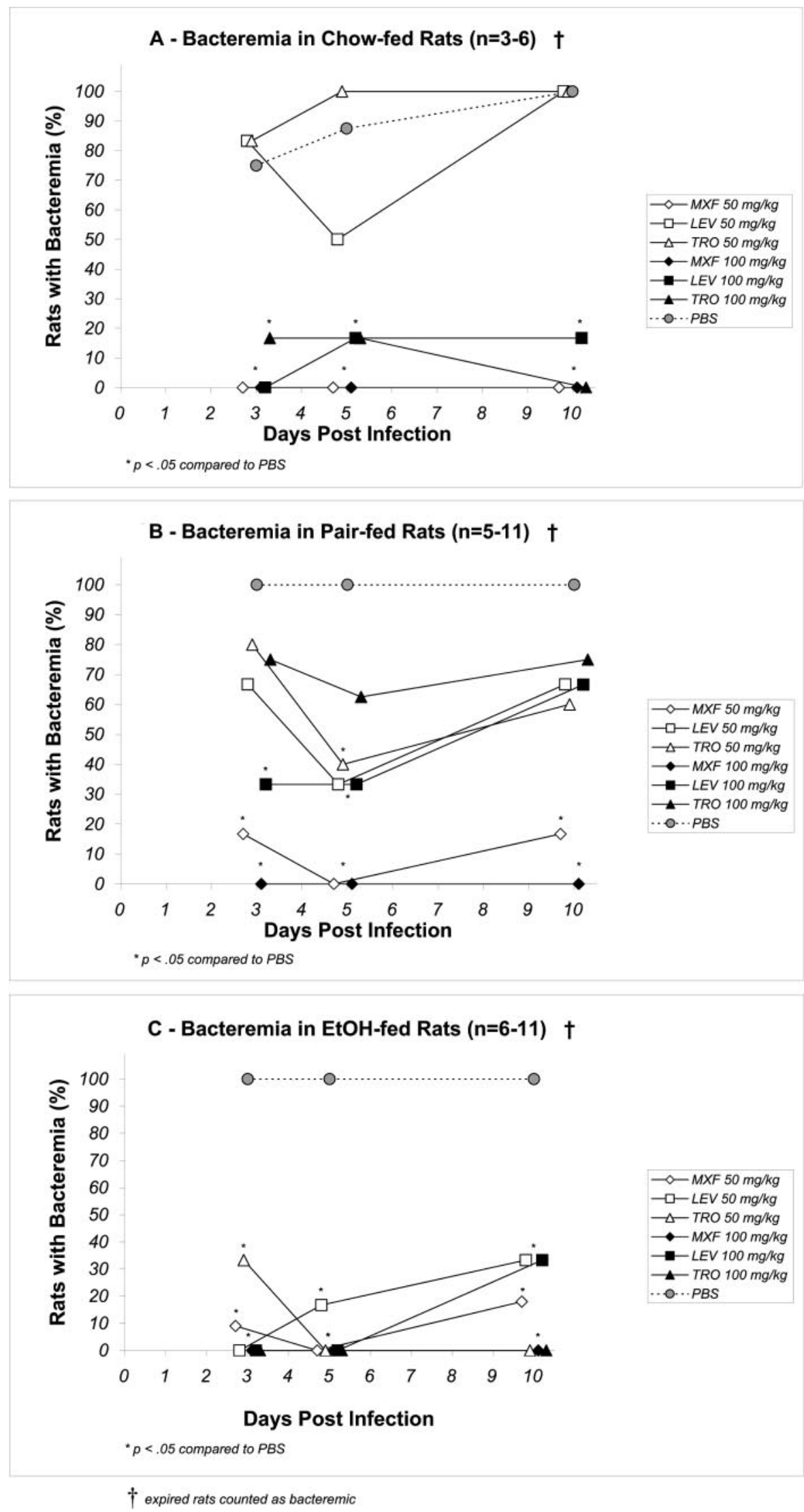

FIG. 3. Cumulative percent bacteremia over 10 days of pneumococcal bacteremia after 5 days of antibiotic treatment in chow-fed (A), pair-fed (B), and EtOH-fed (C) rats. All antibiotics were administered at doses of 50 and $100 \mathrm{mg} / \mathrm{kg} / \mathrm{day}$. LEV, levofloxacin; MXF, moxifloxacin; TRO, trovafloxacin. $*, P<0.05$ compared to the results obtained with PBS. 


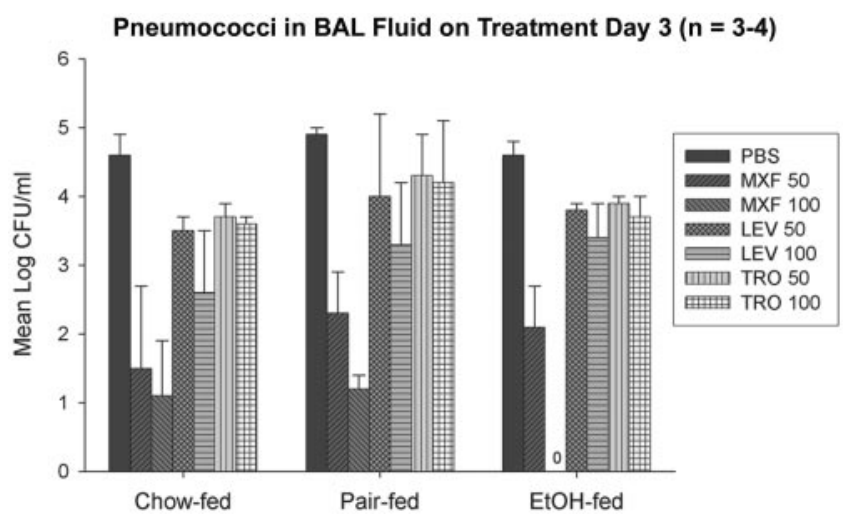

FIG. 4. Mean $\log \mathrm{CFU} / \mathrm{ml}$ of Streptococcus pneumoniae in bronchoalveolar lavage fluid 3 days after the initiation of antibiotic therapy. All antibiotics were administered at doses of 50 and $100 \mathrm{mg} / \mathrm{kg} /$ day. LEV, levofloxacin; MXF, moxifloxacin; TRO, trovafloxacin. The data represent means \pm standard deviations. $P$ was $<0.05$ for moxifloxacin 50 and $100 \mathrm{mg} / \mathrm{kg} /$ day versus PBS in each group.

rats receiving either dose of levofloxacin, two of six (33\%) were still bacteremic at day 10. In the pair-fed rats, moxifloxacin was the most effective in clearing bacteremia compared to the effectiveness of levofloxacin or trovafloxacin at either dose (Fig. 3B). At least four of six $(67 \%)$ of pair-fed rats receiving either levofloxacin or trovafloxacin remained bacteremic at day 10 , regardless of the dose.

WBC, PMNL, and bacterial counts in BAL fluid. On day 3, the mean $S$. pneumoniae count in BAL fluid was at least 1.5 to 2 logs lower for rats receiving moxifloxacin than for rats receiving the other quinolone groups, regardless of the dose or the feeding group $(P<0.05)$ (Fig. 4). Only EtOH-fed rats that received moxifloxacin $100 \mathrm{mg} / \mathrm{kg} /$ day had sterile BAL fluid 3 days following the initiation of treatment. The mean percentage of PMNLs and total WBCs recovered from BAL fluid correlated with the survival data. Among all fluoroquinolonetreated groups, the EtOH-fed rats that experienced the highest survival also had the lowest percentage of PMNLs and the lowest WBC counts. Pair-fed rats had the highest WBC counts and the highest percentage of PMNLs. Although the mean percentage of PMNL and numbers of WBC recovered in BAL fluid varied between feeding groups, the differences were not statistically different (data not shown).

Peripheral WBC and differential cell counts. Mean peripheral WBC and individual cell counts were similar on all measured days of therapy for all feeding groups receiving any antibiotic (data not shown).

Pharmacokinetic and pharmacodynamic parameters. The mean serum $C_{\max }(\mu \mathrm{g} / \mathrm{ml})$ and the mean AUC were the greatest for all antibiotics in the EtOH-fed rats. Moxifloxacin achieved significantly higher $C_{\max }$ values than the other antibiotics $(P<0.05)$ (Table 1$)$. The EtOH-fed group had a lower percent protein binding that resulted in an increased antibiotic free fraction. The calculated $C_{\max } / \mathrm{MIC}$ and $\mathrm{fAUC} / \mathrm{MIC}$ ratios were also highest among the EtOH-fed group for all antibiotics compared to those for the pair-fed and the chow-fed rats. Moxifloxacin had higher a $C_{\max } / \mathrm{MIC}$ and a higher fAUC/MIC than both levofloxacin and trovafloxacin, although this was attributed to lower MICs rather than superior $C_{\max } \mathrm{s}$ and
AUCs. Pair-fed and chow-fed rats receiving $50 \mathrm{mg} / \mathrm{kg} /$ day of trovafloxacin had fAUC/MIC ratios less than 40, which is consistent with the higher mortality among these groups. Levofloxacin followed the same pattern for all groups except the pair-fed group, with a mean fAUC/MIC of 41.2. Moxifloxacin had higher BAL fluid concentrations than either levofloxacin or trovafloxacin, but the differences reached significance only in the EtOH-fed group $(P<0.05)$.

\section{DISCUSSION}

Individuals who abuse EtOH are at increased risk for severe pneumococcal infections (37). The critical interaction of this pathogen begins in the respiratory tree, followed by rapid proliferation in alveolar spaces. EtOH impairs extracellular defense systems that normally facilitate the rapid clearance of bacteria from the respiratory tract (44). EtOH-fed rats are more susceptible to pneumococcal infections and demonstrate decreased PMNL and bactericidal activities (26, 42). Moxifloxacin, a newer "respiratory" fluoroquinolone, has outstanding in vitro activity against $S$. pneumoniae $(4,8,10,31)$. We used our pneumococcal pneumonia model to compare moxifloxacin to levofloxacin and trovafloxacin, quinolones that also have excellent activities against pneumococci. Levofloxacin is predominantly renally eliminated, while both moxifloxacin and trovafloxacin undergo hepatic metabolism and have minimal excretion as unchanged drug. The impact of EtOH on the efficacies of antibiotics that have significant hepatic elimination is unknown. Trovafloxacin was as effective as ceftriaxone or azithromycin in a rat cirrhosis model of pneumococcal infection, but treatment with this antibiotic resulted in a significantly slower normalization of peripheral white blood cell counts (45). In the current study, we chose two different dosage regimens designed to achieve pharmacodynamic ratios for the lower dose just above the reported therapeutic threshold of clinical response.

As predicted, EtOH-fed rats had greater susceptibilities to pneumococcal infection. This was demonstrated by the equivalent mortality of untreated EtOH-fed rats given a 10 -fold lower inoculum compared to that given to the chow-fed or pair-fed nutritional controls. This finding is due to the EtOHinduced defects in the host defense mechanisms against S. pneumoniae $(13,22,26,27,36,42,43,44)$. All antibiotics in both the $50-$ and the $100-\mathrm{mg} / \mathrm{kg} /$ day regimens were equally effective at improving survival in the EtOH-fed rats. Whether these antibiotics could protect this group from the higher inoculum given to the chow-fed and pair-fed control groups remains to be determined. In contrast to what occurred in the EtOH-fed animals, both levofloxacin and trovafloxacin at the $50-\mathrm{mg} / \mathrm{kg} /$ day dose were ineffective in chow-fed rats. In pairfed rats, an increase in the dose from 50 to $100 \mathrm{mg} / \mathrm{kg} /$ day improved survival from $80 \%$ to $100 \%$ among the moxifloxacintreated rats. The higher dose did not improve survival in levofloxacin-treated rats and was associated with increased mortality in rats receiving trovafloxacin. Any variance in the results for the chow-fed and the pair-fed groups is most likely related to nutritional differences in their diets, although comparable results were seen for the two groups in an earlier study (46).

The results of the bacteremia study paralleled the findings in our mortality experiments. Rats receiving levofloxacin and 
TABLE 1. Values of pharmacokinetic and pharmacodynamic parameters for levofloxacin, moxifloxacin, and trovafloxacin following administration of 50 and $100 \mathrm{mg} / \mathrm{kg} /$ day for 5 days in EtOH-fed, pair-fed, and chow-fed rats with pneumococcal pneumonia

\begin{tabular}{|c|c|c|c|c|c|c|}
\hline \multirow{2}{*}{$\begin{array}{l}\text { Group and } \\
\text { parameter }\end{array}$} & \multicolumn{2}{|c|}{ Levofloxacin } & \multicolumn{2}{|c|}{ Moxifloxacin $^{e}$} & \multicolumn{2}{|c|}{ Trovafloxacin } \\
\hline & $50 \mathrm{mg} / \mathrm{kg}$ & $100 \mathrm{mg} / \mathrm{kg}$ & $50 \mathrm{mg} / \mathrm{kg}$ & $100 \mathrm{mg} / \mathrm{kg}$ & $50 \mathrm{mg} / \mathrm{kg}$ & $100 \mathrm{mg} / \mathrm{kg}$ \\
\hline \multicolumn{7}{|l|}{ Ethanol fed } \\
\hline Serum $C_{\max }(\mu \mathrm{g} / \mathrm{ml})$ & $8.1 \pm 3.0$ & $12.2 \pm 3.0$ & $15.1 \pm 6.6$ & $22 \pm 8.6$ & $7.7 \pm 1.5$ & $13.4 \pm 3.7$ \\
\hline $\operatorname{AUC}_{0-24}(\mu \mathrm{g} \cdot \mathrm{h} / \mathrm{ml})$ & $44.9 \pm 14.4$ & $63 \pm 16.6$ & $39.6 \pm 9.7$ & $75.2 \pm 14.3$ & $36.8 \pm 10.8$ & $69.9 \pm 16.1$ \\
\hline fAUC $(\mu \mathrm{g} \cdot \mathrm{h} / \mathrm{ml})$ & $37.3 \pm 11.9$ & $52.3 \pm 13.7$ & $21.9 \pm 5.4$ & $41.6 \pm 7.9$ & $12.9 \pm 3.4$ & $24.5 \pm 5.6$ \\
\hline ELF vol $(\mu \mathrm{g} / \mathrm{ml})$ & $1.2 \pm 0.6$ & $2.5 \pm 1.0$ & $3.9 \pm 1.3$ & $7.3 \pm 1.7$ & $1.9 \pm 0.9$ & $2.7 \pm 1.4$ \\
\hline WBC $(\mu \mathrm{g} / \mathrm{ml})^{a}$ & $3.1 \pm 2.2$ & $5.9 \pm 3.0$ & $4.2 \pm 1.9$ & $6.9 \pm 3.7$ & $1.9 \pm 1.2$ & $3.8 \pm 2.4$ \\
\hline $\mathrm{PB}^{b}(\%)$ & 17.2 & & 44.7 & & 64.9 & \\
\hline$C_{\max } / \mathrm{MIC}^{c}$ & $6.7 \pm 2.4$ & $10.1 \pm 2.5$ & $43.9 \pm 19.2$ & $64.0 \pm 24.9$ & $5.7 \pm 1.2$ & $18.8 \pm 5.2$ \\
\hline $\mathrm{AUC} \mathrm{MIC}^{d}$ & $37.3 \pm 11.9$ & $52.3 \pm 13.7$ & $115.3 \pm 28.2$ & $218.9 \pm 41.6$ & $51.7 \pm 15.2$ & $98.1 \pm 22.6$ \\
\hline $\mathrm{CL}(\mathrm{ml} / \mathrm{min} / \mathrm{kg})$ & $21.1 \pm 1.8$ & $29.2 \pm 1.2$ & $11.6 \pm 0.8$ & $16.9 \pm 1.0$ & $12.2 \pm 1.3$ & $18.9 \pm 1.1$ \\
\hline \multicolumn{7}{|l|}{ Pair fed } \\
\hline Serum $C_{\max }(\mu \mathrm{g} / \mathrm{ml})$ & $7.5 \pm 3.0$ & $12.4 \pm 4.2$ & $10.0 \pm 5.8$ & $17.5 \pm 7.1$ & $7.0 \pm 1.5$ & $9.8 \pm 3.9$ \\
\hline $\operatorname{AUC}_{0-24}(\mu \mathrm{g} \cdot \mathrm{h} / \mathrm{ml})$ & $34.2 \pm 11.9$ & $57.7 \pm 17.2$ & $32.7 \pm 12.9$ & $65.3 \pm 15.4$ & $31.0 \pm 11.2$ & $51.9 \pm 17.9$ \\
\hline fAUC $(\mu \mathrm{g} \cdot \mathrm{h} / \mathrm{ml})$ & $24.4 \pm 8.5$ & $41.2 \pm 12.3$ & $12.5 \pm 4.9$ & $24.9 \pm 5.9$ & $5.8 \pm 2.1$ & $9.7 \pm 3.3$ \\
\hline ELF vol $(\mu \mathrm{g} / \mathrm{ml})$ & $1.9 \pm 0.8$ & $2.7 \pm 0.8$ & $2.1 \pm 1.9$ & $3.7 \pm 1.2$ & $1.7 \pm 0.8$ & $2.9 \pm 1.1$ \\
\hline WBC $(\mu \mathrm{g} / \mathrm{ml})$ & $2.1 \pm 1.4$ & $3.7 \pm 2.1$ & $3.9 \pm 2.0$ & $5.0 \pm 3.3$ & $1.3 \pm 1.0$ & $3.1 \pm 1.8$ \\
\hline $\mathrm{PB}(\%)$ & 28.6 & & 61.9 & & 81.4 & \\
\hline$C_{\max } / \mathrm{MIC}$ & $5.4 \pm 2.1$ & $8.9 \pm 3.0$ & $20.6 \pm 11.9$ & $35.1 \pm 14.2$ & $5.9 \pm 1.1$ & $7.3 \pm 2.9$ \\
\hline AUC/MIC & $24.4 \pm 8.5$ & $41.2 \pm 12.3$ & $67.3 \pm 26.5$ & $130.9 \pm 31.3$ & $23.1 \pm 9.4$ & $38.6 \pm 13.3$ \\
\hline $\mathrm{CL}(\mathrm{ml} / \mathrm{min} / \mathrm{kg})$ & $22.3 \pm 0.9$ & $28.1 \pm 1.2$ & $12.7 \pm 0.7$ & $17.3 \pm 1.1$ & $11.8 \pm 1.0$ & $14.5 \pm 0.9$ \\
\hline \multicolumn{7}{|l|}{ Chow fed } \\
\hline Serum $C_{\max }(\mu \mathrm{g} / \mathrm{ml})$ & $6.6 \pm 1.5$ & $9.3 \pm 3.7$ & $10.6 \pm 1.7$ & $14.5 \pm 4.8$ & $6.1 \pm 2.1$ & $11.9 \pm 3.9$ \\
\hline $\operatorname{AUC}_{0-24}(\mu \mathrm{g} \cdot \mathrm{h} / \mathrm{ml})$ & $35.7 \pm 13.3$ & $54.9 \pm 17.1$ & $34.8 \pm 12.1$ & $67.9 \pm 15.7$ & $28.9 \pm 8.9$ & $52.7 \pm 14.8$ \\
\hline fAUC $(\mu \mathrm{g} \cdot \mathrm{h} / \mathrm{ml})$ & $25.4 \pm 9.4$ & $39.0 \pm 12.1$ & $14.1 \pm 4.9$ & $27.4 \pm 6.4$ & $4.9 \pm 1.5$ & $8.9 \pm 2.5$ \\
\hline ELF vol $(\mu \mathrm{g} / \mathrm{ml})$ & $2.0 \pm 0.6$ & $2.9 \pm 1.0$ & $3.4 \pm 1.3$ & $5.9 \pm 1.5$ & $1.9 \pm 0.8$ & $2.7 \pm 0.6$ \\
\hline WBC $(\mu \mathrm{g} / \mathrm{ml})$ & $2.5 \pm 1.1$ & $4.2 \pm 2.9$ & $3.5 \pm 1.7$ & $4.6 \pm 2.2$ & $1.4 \pm 0.9$ & $3.2 \pm 1.4$ \\
\hline PB (\%) & 29.0 & & 59.5 & & 83.1 & \\
\hline$C_{\max } / \mathrm{MIC}$ & $4.7 \pm 1.1$ & $6.6 \pm 2.6$ & $22.6 \pm 3.6$ & $30.9 \pm 10.2$ & $4.1 \pm 1.6$ & $8.1 \pm 2.6$ \\
\hline AUC/MIC & $25.4 \pm 9.4$ & $39.0 \pm 12.1$ & $74.2 \pm 25.8$ & $144.7 \pm 33.5$ & $19.5 \pm 6.0$ & $35.6 \pm 10.0$ \\
\hline $\mathrm{CL}(\mathrm{ml} / \mathrm{min} / \mathrm{kg})$ & $22.9 \pm 1.4$ & $29.7 \pm 1.3$ & $12.3 \pm 0.9$ & $16.2 \pm 1.0$ & $12.8 \pm 1.2$ & $15.9 \pm 1.4$ \\
\hline
\end{tabular}

${ }^{a}$ White blood cells from BAL fluid.

${ }^{b} \mathrm{~PB}$, protein binding of the antibiotic in percent.

${ }^{c}$ Calculated by using the antibiotic free fraction.

${ }^{d}$ Calculated by using the antibiotic free fraction.

${ }^{e}$ Moxifloxacin Free AUC/MIC and $C_{\max } / \mathrm{MIC}$ ratios for moxifloxacin were significantly greater than those for either levofloxacin and trovafloxacin at equivalent doses $(P<0.05)$.

trovafloxacin at the $50-\mathrm{mg} / \mathrm{kg}$ dose were more likely to be bacteremic at all time periods during and 5 days after the end of antibiotic therapy. Some rats in the EtOH-fed group either remained bacteremic through therapy, or after a period of negative cultures, regrowth occurred after the discontinuation of antimicrobial therapy. Persistent or recurrent bacteremia was observed with 50 and $100 \mathrm{mg} / \mathrm{kg} /$ day of levofloxacin as well as $50 \mathrm{mg} / \mathrm{kg}$ of moxifloxacin.

The pharmacodynamic features of individual antibiotics may explain, in part, the results observed in this study. The effectiveness of fluoroquinolone antibiotics has been correlated with $\mathrm{AUC} / \mathrm{MIC}$ and $C_{\max } / \mathrm{MIC}$ ratios $(7,32,38,59)$. More recently, the ratios determined with the unbound or free fraction of the drug appear to have a stronger correlation with effectiveness $(38,59)$. Although quinolone pharmacodynamic parameters are likely organism specific, studies suggest that the fAUC/MIC ratios should exceed 30 for $S$. pneumoniae infections $(38,59)$. In animal and human antibiotic studies of pharmacodynamics, pharmacokinetic variables and protein binding characteristics may contribute to efficacy. Our study did not show statistical differences in the AUC or $C_{\max }$ values among any antibiotic or feeding regimens. However, the EtOH-fed group demonstrated higher fAUC/MIC ratios for all compounds, with the values exceeding 50 with both dosage regimens. Of note, the EtOH-fed group also responded better than the diet control groups to treatment with levofloxacin and trovafloxacin and was the only group in which levofloxacin and trovafloxacin demonstrated efficacies comparable to that of moxifloxacin at the 50$\mathrm{mg} / \mathrm{kg}$ dose. In contrast, levofloxacin and trovafloxacin $\mathrm{AUC}_{0-24} \mathrm{~S}$ were consistently less than 40 and the fAUC/MIC ratio was $<30$ in both the pair-fed and the chow-fed animals, the groups which also had higher mortality rates.

It is well accepted that only free, unbound antibiotic can readily transfer from the central vascular compartment to various tissues and body compartments. The degree of binding is a predictor of extravascular distribution (6). In this study, the primary contributor to changes in the fAUC/MIC ratios appeared to be a decrease in protein binding observed in the EtOH-fed group. The pair- and chow-fed groups demonstrated levels of protein binding comparable to those observed in pharmacokinetic studies with noninfected rats. The cause for these alterations in protein binding remains unknown. Because 
the rats only had 2 weeks of EtOH exposure, it is unlikely that hepatic disease induced a reduction in serum albumin. In this model, serum proteins begin to fall only after continuous exposure to EtOH for at least 5 weeks (16). Also, the EtOH-fed rats developed modest increases only in liver transaminases. It is unlikely that the findings were due to an alteration in hepatic metabolism because trovafloxacin (hepatic elimination) demonstrated results similar to those for the renally eliminated drug levofloxacin. Moxifloxacin, which also undergoes hepatic elimination, was superior to both levofloxacin and trovafloxacin. Because the $S$. pneumoniae MIC, AUCs, and serum proteins remained constant from one group to the next, it appears that some other endogenous or exogenous factor altered the percent bound drug in the EtOH-fed group. EtOH may have decreased the affinity of albumin for each of the quinolones, with a resultant increase in the free fraction. Limited data are available regarding the effect of hepatic dysfunction on quinolone pharmacokinetics. A previous study with a hepatically eliminated fluoroquinolone in patients with mild to moderate hepatic impairment demonstrated increases in AUC (18). In humans, levofloxacin may be effective despite the protein binding, because it achieves higher intrapulmonary concentrations (11). It is unlikely that trovafloxacin, despite its low MICs, would be able to overcome the high protein binding in view of its low pulmonary BAL fluid concentrations (2).

Rats with fAUC/MIC ratios $<40$ demonstrated a higher mortality than those with ratios $>50$. Although the latter ratio is consistent with those observed in different animal models by Craig and colleagues, they do call into question AUC/MIC ratios between 30 and 40 (32). Generally, animal pharmacodynamic studies have shown that fAUC/MIC ratios $<30$ are associated with a $>50 \%$ mortality rate in experimental models of pneumonia. The AUC/MIC ratio is a function of antibiotic pharmacokinetics, the protein binding, and the MIC of the infecting organism. Moxifloxacin and trovafloxacin had similar MICs for the pneumococcal strain used and achieved similar AUCs. However, trovafloxacin consistently yielded lower fAUC/MIC ratios, which could explain the higher mortality in this treatment group. The pharmacodynamic profile of moxifloxacin is generally regarded as equivalent or superior to those of all commercially available fluoroquinolones when community-acquired pathogens like the pneumococcus are considered (14). The fAUC/MIC ratios observed for moxifloxacin are similar to those reported in studies with humans $(14,15)$. In a comparative analysis of 12 antimicrobial agents against respiratory pathogens, moxifloxacin possessed the lowest MICs toward S. pneumoniae in comparison to those of the other available quinolones (8). The pharmacokinetics of levofloxacin, including the fAUC observed in this rat model, are similar to those extrapolated from human studies $(7,59)$. The two factors that appeared to affect the efficacy of levofloxacin were the MIC of the infecting pathogen and the changes in protein binding (49). In the chow-fed and the pair-fed rats, the protein binding rose and the AUC/MIC ratios subsequently dropped below 30, resulting in higher mortality rates. AUC/MIC calculations by using the AUC from total drug concentrations would have resulted in values above the efficacy threshold of 30 at all doses and for all groups for both levofloxacin and trovafloxacin. These data confirm the use of the free fractions for AUC/
MIC calculations and call into question the threshold value of 30 when total drug is used.

We chose to use an S. pneumoniae isolate with MICs similar to those observed in the community. Among over 4,900 isolates tested in the United States in 2001 and 2002, the levofloxacin MIC at which $90 \%$ of isolates are for S. pneumoniae was $1.0 \mu \mathrm{g} / \mathrm{ml}$ (49). We used a pathogen with this same MIC for levofloxacin. In our rat model, treatment failure with levofloxacin was correlated with suboptimal fAUC/MIC ratios. These results are similar to those in other animal studies of pneumonia that have evaluated the pharmacodynamics and efficacy of levofloxacin $(7,12,15,40)$. In our model, higher doses of levofloxacin resulted in higher fAUC/MIC ratios and gave levofloxacin efficacy comparable to that of moxifloxacin. Levofloxacin was shown to be effective in patients with CAP (17). High-dose, short-course therapy with a dose of $750 \mathrm{mg}$ /day enhanced the pharmacodynamic parameters for levofloxacin and produced excellent microbiological eradication and clinical cure rates (17).

In conclusion, moxifloxacin at doses equivalent to those of levofloxacin and trovafloxacin resulted in the highest fAUC/ MIC ratios and was more effective than levofloxacin and trovafloxacin in our rat model of pneumococcal pneumonia. EtOH increased the free fraction of all antibiotics and allowed the levofloxacin and trovafloxacin AUC/MIC ratios to increase to values $>50$, resulting in efficacy equivalent to that observed with moxifloxacin. Chow-fed and pair-fed animals demonstrated increased protein binding and fAUC/MIC ratios $<30$ that contributed to their higher mortality rates. The conventional breakpoints of an AUC/MIC ratio of 30 to 40 by use of the total drug for determination of the ratio appear in question for levofloxacin and trovafloxacin in this model. It appears that the achievement of an adequate pharmacodynamic parameter, the fAUC/MIC ratio, is a more important predictor of successful treatment than any immune defects that $\mathrm{EtOH}$ induces. These data should be validated with clinical trials with humans who abuse EtOH and develop pneumococcal pneumonia.

\section{ACKNOWLEDGMENTS}

This work was supported in part by a grant from Bayer, Inc., and by a Merit Review grant from the U.S. Department of Veterans Affairs (to L. C. Preheim and M. Gentry-Nielsen).

We thank Dennis W. Wolff for his able assistance with computer graphics.

\section{REFERENCES}

1. American Thoracic Society. 2001. Guidelines for the management of adults with community-acquired pneumonia. Am. J. Respir. Crit. Care Med. 163: 1730-1754.

2. Andrews, J. M., D. Honebourne, N. P. Brenwald, D. Bannerjee, M. Iredale, B. Cunningham, and R. Wise. 1997. Concentrations of trovafloxacin in bronchial mucosa, epithelial lining fluid, alveolar macrophages and serum after administration of single or multiple oral doses to patients undergoing fibreoptic bronchoscopy. J. Antimicrob. Chemother. 39:797-802.

3. Baldwin, D. R., D. Honeybourne, and R. Wise. 1992. Pulmonary disposition of antimicrobial agents: methodological considerations. Antimicrob. Agents Chemother. 36:1171-1175.

4. Barman Balfour J. A., and L. R. Wiseman. 1999. Moxifloxacin. Drugs 57: 363-373.

5. Bartlett, J. G., S. F. Dowell, L. A. Mandell, T. M. File, Jr., D. M. Musher, and M. J. Fine. 2000. Update of practice guidelines for the management of community-acquired pneumonia in adults. Clin. Infect. Dis. 31:347-382.

6. Bergogne-Bérézin, E. 2002. Clinical role of protein binding of quinolones. Clin. Pharmacokinet. 41:741-750. 
7. Berry, V., R. Page, J. Satterfield, C. Singley, C. R. Straub, and G. Woodnutt. 2000. Comparative in vivo activity of gemifloxacin in a rat model of respiratory tract infection. J. Antimicrob. Chemother. 45(Suppl. S1):79-85.

8. Blondeau, J. M. 1999. A review of the comparative in-vitro activities of 12 antimicrobial agents, with a focus on five new respiratory quinolones. J. Antimicrob. Chemother. 43(Suppl. B):1-11.

9. Brighty, K. E., and T. D. Gootz. 1997. The chemistry and biological profile of trovafloxacin. J. Antimicrob. Chemother. 39(Suppl. B):1-14.

10. Buxbaum, A., U. Straschil, C. Moser, W. Graninger, A. Georgopoulos, et al 1999. Comparative susceptibility to penicillin and quinolones of 1385 Streptococcus pneumoniae isolates. J. Antimicrob. Chemother. 43(Suppl. B):13-18.

11. Capitano, B., H. M. Mattoes, E. Shore, A. O'Brien, C. Sutherland, and D. P. Nicolau. 2004. Steady state intrapulmonary concentrations of moxifloxacin, levofloxacin, and azithromycin in older adults. Chest 125:965-973.

12. Cheng, F. C., T. R. Tsai, Y. F. Chen, L. C. Hung, and T. H. Tsai. 2002 Pharmacokinetic study of levofloxacin in rat blood and bile by microdialysis and high-performance liquid chromatography. J. Chromatogr. A 961:131136.

13. Cooper, B., and E. G. Maderazo. 1988. Alcohol abuse and impaired immunity. Infect. Med. 5:479-487.

14. Culley, C. M., M. K. Lacy, N. Klutman, and B. Edwards. 2001. Moxifloxacin clinical efficacy and safety. Am. J. Health-Syst. Pharm. 58:379-388.

15. Davis, R., and H. M. Bryson. 1994. Levofloxacin: a review of its antibacterial activity, pharmacokinetics, and therapeutic efficacy. Drugs 47:677-700.

16. DeCarli, M., and C. S. Lieber. 1967. Fatty liver in the rat after prolonged intake of ethanol with a nutritionally adequate new liquid diet. J. Nutr. 91:331-336.

17. Dunbar, L. M., R. G. Wunderink, M. P Habib, L. G. Smith, A. M. Tenneberg, M. M. Khashab, B. A. Wiesinger, J. X. Xiang, N. Zadeikis, and J. B. Kahn. 2003. High-dose, short-course levofloxacin for community-acquired pneumonia: a new treatment paradigm. Clin. Infect. Dis. 37:752-760.

18. Efthymiopoulos, C., S. L. Bramer, A. Maroli, J. F. Flaherty, Jr., E. Wolfe, N. Bass, and K. Somberg. 1997. Grepafloxacin pharmacokinetics in individuals with hepatic dysfunction. Clin. Pharmacokinet. 33(Suppl. 1):25-31.

19. Eliopoulos G. M., K. Klimm, C. T. Eliopoulos, M. J. Ferraro, and R. C Moellering. Jr. 1993. In vitro activity of CP-99,219, a new fluoroquinolone, against clinical isolates of gram-positive bacteria. Antimicrob. Agents Chemother. 37:366-370.

20. Ellbogen, M. H., K. M. Olsen, M. J. Gentry-Nielsen, and L. C. Preheim. 2003. Efficacy of liposome-encapsulated ciprofloxacin compared with ciprofloxacin and ceftriaxone in a rat model of pneumococcal pneumonia. J. Antimicrob. Chemother. 51:83-91.

21. File, T. M., Jr. 2003. Community acquired pneumonia. Lancet 361:19912001.

22. Gentry, M. J., and L. C. Preheim. 1999. Models of pneumonia in ethanoltreated rats, p. 501-507. In O. Zak and M. Sande (ed.), Handbook of animal models of infection. Academic Press, Inc., San Diego, Calif.

23. Gentry, M. J., M. U. Snitily, and L. C. Preheim. 1996. Decreased uptake and killing of Streptococcus pneumoniae within the lungs of cirrhotic rats. Immunol. Infect. Dis. 6:43-47.

24. Gentry-Nielsen, M. J., K. M. Olsen, and L. C. Preheim. 2002. Pharmacodynamic activity and efficacy of linezolid in a rat model of pneumococcal pneumonia. Antimicrob. Agents Chemother. 46:1345-1351.

25. Hook, E. W., C. A. Horton, and D. R. Schaberg. 1983. Failure of intensive care unit support to influence mortality from pneumococcal bacteremia. JAMA 249:1055-1057.

26. Jareo, P. W., L. C. Preheim, P. D. Lister, and M. J. Gentry. 1995. The effect of ethanol ingestion on killing of Streptococcus pneumoniae, Staphylococcus aureus, and Staphylococcus epidermidis rat neutrophils. Alcohol Alcoholism 30:311-318.

27. Jareo, P. W., L. C. Preheim, and M. J. Gentry. 1996. Ethanol ingestion impairs neutrophil bactericidal mechanisms against Streptococcus pneumoniae. Alcohol. Clin. Exp. Res. 20:1646-1652.

28. Jorgensen, J. H., L. M. Weigel, M. J. Ferraro, J. M. Swenson, and F. C. Tenover. 1999. Activities of newer fluoroquinolones against Streptococcus pneumoniae clinical isolates including those with mutations in the $\operatorname{gyr} A, \operatorname{parC}$ and parE loci. Antimicrob. Agents Chemother. 43:329-334.

29. Kapp, A., and G. Zeck-Kapp. 1990. Activation of the oxidative metabolism in human polymorphonculear neutrophilic granulocytes: the role of immunomodulating cytokines. J. Investig. Dermatol. 95:94S-99S.

30. Kim, Y. S., Q. Liu, L. L. Chow, and M. G. Täuber. 1997. Trovafloxacin in treatment of rabbits with experimental meningitis caused by high-level penicillin-resistant Streptococcus pneumoniae. Antimicrob. Agents Chemother. 41:1186-1189.

31. Krasemann, C., J. Meyer, and G. Tillotson. 2001. Evaluation of the clinical microbiology profile of moxifloxacin. Clin. Infect. Dis. 32(Suppl. 1):S51-S63.

32. Leggett, J. E., B. Fantin, S. Ebert, K. Totsuka, B. Vogelman, W. Calame, H Mattie, and W. A. Craig. 1989. Comparative antibiotic dose-effect relations at several dosing intervals in murine pneumonitis and thigh infection models. J. Infect. Dis. 159:281-292.
33. Lieber, C. S. 1991. Hepatic, metabolic and toxic effects of ethanol: 1991 update. Alcohol. Clin. Exp. Res. 15:573-592

34. Litwin, S. D. 1990. Alcohol research in the Veterans Administration: a success story. Alcohol. Clin. Exp. Res. 14:787-788.

35. Mandell, L. A., J. G. Bartlett, S. F. Dowell, T. M. File, Jr., D. M. Musher, and C. Whitney. 2003. Update of practice guidelines for the management of community-acquired pneumonia in immunocompetent adults. Clin. Infect. Dis. 37:1405-1433.

36. Mellencamp, M. A., and L. C. Preheim. 1991. Pneumococcal pneumonia in a rat model of cirrhosis: effects of cirrhosis on pulmonary defense mechanisms against Streptococcus pneumoniae. J. Infect. Dis. 163:102-108.

37. Mufson, E. O., D. M. Kruss, R. E. Wasil, and W. I. Metzger. 1974. Capsular types and outcome of bacteremic pneumococcal disease in the antibiotic era. Arch. Intern. Med. 134:505-510.

38. Nicolau, D. P., H. M. Mattoes, M. Banevicius, D. Xuan, and C. H. Nightingale. 2003. Pharmacodynamics of a novel des-F(6)-quinolone, BMS-284756, against Streptococcus pneumoniae in the thigh infection model. Antimicrob. Agents Chemother. 47:1630-1635.

39. Olsen, K. M., G. S. San Pedro, L. P. Gann, P. O. Gubbins, D. M. Halinski, and G. D. Campbell. 1996. Intrapulmonary pharmacokinetics of azithromycin in healthy volunteers given five oral doses. Antimicrob. Agents Chemother. 40:2582-2585.

40. Perry, A. C., B. Prpa, M. S. Rouse, K. E. Piper, A. D. Hanssen, J. M. Steckelberg, and R. Patel. 2003. Levofloxacin and trovafloxacin inhibition of experimental fracture-healing. Clin. Orthop. 414:95-100.

41. Preheim, L. C., and M. J. Gentry. 1996. Infection in the alcoholic, p. 308311. In D. Schlossberg (ed.), Current therapy of infectious disease. The C.V. Mosby Co., St. Louis, Mo.

42. Preheim, L. C., M. J. Gentry, and M. U. Snitily. 1991. Pulmonary recruitment, adherence, and chemotaxis of neutrophils in a rat model of cirrhosis and pneumococcal pneumonia. J. Infect. Dis. 164:1203-1206.

43. Preheim, L. C., M. A. Mellencamp, M. U. Snitily, and M. J. Gentry. 1992. Effect of cirrhosis on the production and efficacy of pneumococcal capsular antibody in a rat model. Am. Rev. Respir. Dis. 146:1054-1058.

44. Preheim, L. C., M. U. Snitily, and M. J. Gentry. 1996. Effects of granulocyte colony-stimulating factor in cirrhotic rats with pneumococcal pneumonia. J. Infect. Dis. 174:225-228.

45. Preheim, L. C., K. M. Olsen, M. Yue, M. U. Snitily, and M. J. GentryNielsen. 2005. Effect of cirrhosis on antibiotic efficacy in a rat model of pneumococcal pneumonia. Diagn. Microbiol. Infect. Dis. 51:103-111.

46. Preheim, L. C., K. M. Olsen, M. Yue, M. U. Snitily, and M. J. Gentry. 1999. Ethanol feeding does not affect the efficacy or pharmacokinetics of azithromycin, trovafloxacin, or ceftriaxone in a rat model of pneumococcal pneumonia. Alcohol. Clin. Exp. Res. 23:842-849.

47. Renard, S. I., G. Basset, D. Lecossier, K. M. O'Donnell, P. Pinkston, P. G. Martin, and R. G. Crystal. 1986. Estimation of volume of epithelial lining fluid recovered by lavage using urea as a marker of dilution. J. Appl. Physiol. 69:532-538

48. Sahm, D. F., C. Thornsberry, M. E. Jones, R. S. Blosser, I. A. Critchley, A. T. Evangelista, and J. A. Karlowsky. 2002. Correlations of antimicrobial resistance among Streptococcus pneumoniae in the United States: 2001-2002 TRUST surveillance, abstr. C2-1640. Abstr. Intersci. Conf. Antimicrob. Agents Chemother. American Society for Microbiology, Washington, D.C.

49. Scheld, W. M. 2003. Maintaining fluoroquinolone class efficacy: review of influencing factors. Emerg. Infect. Dis. 9:1-9.

50. Siefert, H. M., C. Kohlsdorfer, W. Steinke, and A. Witt. 1999. Pharmacokinetics of the 8-metheoxyquinolone, moxifloxacin: tissue distribution in male rats. J. Antimicrob. Chemother. 43(Suppl. B):61-67.

51. Snitily, M. U., M. J. Gentry, M. A. Mellencamp, and L. C. Preheim. 1991. A simple method for collection of blood from the rat foot. Lab. Anim. Sci. 41:285-287.

52. Teng, R., G. Girard, T. D. Gootz, T. Foulds, and T. E. Liston. 1996. Pharmacokinetics of trovafloxacin (CP-99,219), a new quinolone, in rats, dogs, and monkeys. Antimicrob. Agents Chemother. 40:561-566.

53. Teng, R., T. G. Tensfeldt, T. E. Liston, and G. Foulds. 1996. Determination of trovafloxacin, a new quinolone antibiotic, in biological samples by reverse-phase high performance liquid chromatography. J. Chromatogr. B 675:53-59.

54. Thomson, K. S., S. A. Chartrand, C. C. Sanders, and S. L. Block. 1997 Trovafloxacin, a new fluoroquinolone with potent activity against Streptococcus pneumoniae. Antimicrob. Agents Chemother. 41:478-480.

55. Vaudaux, P., P. Francois, C. Bisognano, J. Schrenzel, and D. P. Lew. 2002 Comparison of levofloxacin, alatrofloxacin, and vancomycin for prophylaxis and treatment of experimental foreign-body-associated infection by methicillin-resistant Staphylococcus aureus. Antimicrob. Agents Chemother. 46: 1503-1509.

56. Vincent, J., R. Teng, B. A. Baris, S. A. Willavize, R. J. Polzer, and H. L. Friedman. 1997. Pharmacokinetics and safety of trovafloxacin in healthy male volunteers following administration of single intravenous does of the pro-drug, alatrofloxacin. J. Antimicrob. Chemother. 39(Suppl. B):75-80.

57. Weinberg, E. D. 1990. Cellular iron metabolism in health and disease. Drug Metab. Rev. 22:531-579. 
58. Wilcox. M., A. Kervitsky, L. C. Watter, and T. E. King, Jr. 1988. Quantification of cells recovered by bronchoalveolar lavage. Am. Rev. Respir. Dis. 138:74-80.

59. Wright, D. H., G. H. Brown, M. L. Peterson, and J. C. Rotschafer. 2000 Application of fluoroquinolone pharmacodynamics. J. Antimicrob. Chemother. 46:669-683.

60. Wright, D. H., V. K. Herman, F. N. Konstantinides, and J. C. Rotschafer. 1998. Determination of quinolones antibiotics in growth media by reversed- phase high performance liquid chromatography. J. Chromatogr. B Biomed Sci. Appl. 709:97-104.

61. Yu, V. L., C. C. Chiou, C. Feldman, A. Ortqvist, J. Rello, A. J. Morris, L. M. Baddour, C. M. Luna, D. R. Snydman, M. Ip, W. C. Ko, B. F Chedid, A. Andremont, and K. P. Klugman. 2003. An international prospective study of pneumococcal bacteremia: correlation with in vitro resistance, antibiotics administered, and clinical outcome. Clin. Infect. Dis. 37:230-237. 